\title{
Gastrointestinal Manifestations and Liver Injury: Correlation with Mortality and Clinical Outcomes in Patients with COVID-19
}

\author{
Authors: \\ Michael Makar, ${ }^{1}$ Carlos D. Minacapelli,, ${ }^{2}$ Kapil Gupta,, ${ }^{2}$ Abhishek \\ Bhurwal, ${ }^{2}$ You Li, ${ }^{2}$ Carolyn Catalano, ${ }^{2}$ Romy Bareket, ${ }^{3}$ Samuel Jo, ${ }^{1}$ \\ Abhishek A. Chouthai, ${ }^{3}{ }^{*}$ Vinod Rustgi ${ }^{2,4}$ \\ 1. Department of Internal Medicine, Rutgers Robert Wood Johnson Medical School, \\ New Brunswick, New Jersey, USA \\ 2. Department of Medicine, Division of Gastroenterology and Hepatology, Rutgers \\ Robert Wood Johnson Medical School, New Brunswick, New Jersey, USA \\ 3. Rutgers Robert Wood Johnson Medical School, New Jersey, USA \\ 4. Center for Liver Diseases and Masses, Rutgers Robert Wood Johnson School of \\ Medicine, New Brunswick New Jersey, USA \\ *Correspondence to vinod.rustgi@rutgers.edu
}

Disclosure: $\quad$ The authors have declared no conflicts of interest. The study was reviewed and approved for publication by the authors' Institutional Reviewer.

Acknowledgements: All authors contributed to all four criteria, concept and design, data acquisition, statistical analysis, and drafting of manuscript. The authors thank data collection assistance by Vinit Palayekar (MD candidate), Daniel Kats (MD candidate), and Robert Gaffey (MD).

Received:

19.09.20

Accepted:

24.11.20

Keywords:

Coronavirus disease (COVID-19), gastrointestinal (GI) manifestations, intensive care unit (ICU), liver Injury (LI), mortality.

Citation:

EMJ Hepatol. 2021;DOI/10.33590/emjhepatol/20-00233.

\section{Abstract}

Background and aims: Reports indicate that patients with severe acute respiratory syndrome coronavirus 2 (SARS-CoV-2) infection present with gastrointestinal (GI) manifestations and abnormal liver function; however, the impact on clinical findings is unclear. The aim of this study is to report the impact of gastrointestinal and liver injury (LI) associated with coronavirus disease (COVID-19).

Material and methods: The authors retrospectively evaluated patients who presented to the emergency department and were diagnosed with COVID-19 by PCR nasopharyngeal swab. Primary outcomes were the impact of GI findings and LI on in-hospital mortality. Secondary outcomes were length of stay in hospital and need for intensive care unit (ICU) level care.

Results: Of the entire cohort, $64.0 \%$ had LI during hospitalisation. LI was present in $88.7 \%$ of those who died. In multivariate analysis, GI manifestations were associated with decreased risk of mortality (odds ratio [OR]: 0.46; 95\% confidence interval [Cl]: 0.24-0.90; $p=0.002$ ). Gl symptoms did not impact length of stay, 5.06 days for patients with $\mathrm{Gl}$ symptoms versus 4.74 in patients without (OR: 1.01; 95\% Cl: 0.96-1.07; $\mathrm{p}=0.43$ ), or need for ICU (OR: 0.97; 95\% Cl: 0.60-1.58; $\mathrm{p}=0.9076$ ) (Table 1). In multivariate analysis, LI was associated with increased mortality (OR: 8.60; 95\% Cl: 3.49-21.15; 
$\mathrm{p}<0.0001$ ), need for ICU (OR: 10.94; 95\% Cl: 4.07-29.45; $\mathrm{p}<0.0001$ ), and length of stay 5.87 days versus 3.01 days (OR: 1.23; 95\% $\mathrm{Cl}: 1.14-1.32 ; \mathrm{p}<0.0001$ ).

Conclusion: The results of the data analysis show that Gl symptoms may inversely correlate with mortality, while LI is associated with increased mortality, length of stay, and ICU admission.

\section{INTRODUCTION}

Severe acute respiratory syndrome coronavirus 2 (SARS-CoV-2) arose in December 2019 in Wuhan, China, and was noted to cause a severe respiratory distress syndrome termed coronavirus disease (COVID-19). ${ }^{1}$ In March 2020, the World Health Organization (WHO) declared COVID-19 a pandemic and it has subsequently become a global public health and economic threat. ${ }^{2}$ As of September 2020, there were $30,055,710$ confirmed cases and 943,433 confirmed deaths across 216 countries and territories globally. ${ }^{3}$ The full genome sequencing and protein sequence analysis of the COVID-19 virus revealed that it shares $79.5 \%$ sequence identity with SARS-CoV and is classified as a SARS-related coronavirus (CoV). ${ }^{4}$ In the past 20 years, specifically two epidemics caused by CoV have occurred: SARS-CoV in China 20022003 (8,000 cases, 800 deaths, fatality rate of 9.6\%) and the 2012 Middle East respiratory syndrome (MERS)-CoV in Saudi Arabia (2,500 cases, 800 deaths, fatality rate of $35.0 \%$ ).

SARS-CoV-2 is a positive-stranded, enveloped, RNA virus with a nucleocapsid, which belongs to the Coronaviridae family. SARS-CoV-2 is able to produce nonstructural proteins (nsps; nsp 1-16) and structural proteins: envelopes, nucleocapsid proteins, and spikes., ${ }^{1,5}$ The virulence of SARSCoV-2 links to its nsp and structural proteins. The nsps have been hypothesised to play a role in halting innate immunological response, while the structural proteins may play a role in the pathogenicity of SARS-CoV-2 by mediating viral assembly and release. ' The structural spike glycoproteins (subunits S1 and subunit S2) have a receptor binding domain, which acts as a binding site for the human angiotensin converting enzyme 2 receptor (ACE2R). The receptor binding domain spike thereby can bind to ACE2R in expressing tissue.

The ACE2R is part of a circulating hormonal system, the renin-angiotensin system (RAS), which plays a significant role in blood pressure and fluid balance regulation homeostasis. ${ }^{6}$ Organ-specific RAS activity promotes tissue growth, tissue differentiation, and local inflammation. ${ }^{7}$ ACE2R specifically has been found to be expressed in the epithelial tissue of the oral mucosa, lung (Type II alveolar cells), oesophagus (oesophageal epithelial cells), ileum (absorptive enterocytes), colon (absorbtive enterocytes), kidney (proximal tubule cells), and bladder (urothelial bladder cells), and the pancreas. $^{8}$ SARS-CoV-2 enters the lung parenchyma by utilising the Type II alveolar cells and mediates entry by binding to the ACE2R and is subsequently cell free and/or macrophagephagocytosed, and can thereby spread to other organs that may also express the ACE2R, yielding multiorgan injury. ${ }^{4}$ Coronaviruses can cause a broad spectrum of disease, including but not limited to respiratory, enteric, hepatic, and neurological disease. ${ }^{1,4,8,9}$ Clinically, COVID-19 is characterised as either asymptomatic, uncomplicated mild illness (symptoms of upper respiratory infection, malaise, headache, myalgia, anosmia, nausea, vomiting, diarrhoea), moderate-to-severe pneumonia. On the other end of the spectrum, severe COVID-19 can result in respiratory failure and acute respiratory distress syndrome requiring mechanical ventilation, to multiorgan and systemic disease, including sepsis, septic shock, and multiple organ dysunction syndrome.

Currently, literature reviewing COVID-19 demonstrates the multiorgan impact of COVID-19. Gastrointestinal (GI) manifestations and liver injury (LI) are increasingly recognised findings in patients with COVID-19. Initial studies have reported digestive symptoms to be present in approximately $40-50 \%$ of patients. ${ }^{10,11}$ In a meta-analysis of patients with COVID-19, GI symptoms had a prevalence rate of $9.8 \%$ in adults and $9.6 \%$ in children;12 this included diarrhoea, nausea, vomiting, abdominal discomfort, and abdominal pain. SARS-CoV-2 RNA was detected in the faeces of $30.3 \%$ of patients positive for COVID-19.12 Diarrhoea is the most common GI symptom and has been reported in as many as 
$36 \%$ of patients. ${ }^{13}$ However, there is a significant variation in studies regarding the prevalence and impact of Gl symptoms in COVID-19.14 The impact of $\mathrm{Gl}$ symptoms on clinical outcomes is also unclear. Studies have shown an association between abnormal liver enzymes and the severity of COVID-19. ${ }^{9}$ In addition, LI has been associated with adverse clinical outcomes and mortality. ${ }^{15}$

At present, studies are needed to identify the prevalence and clinical impact of $\mathrm{Gl}$ and $\mathrm{LI}$ findings in patients with COVID-19. Therefore, this study reports the prevalence and clinical impact of $\mathrm{Gl}$ and LI associated with COVID-19 in the highly diverse patient population seen in the USA.

\section{MATERIALS AND METHODS}

This is an institutional review board-approved retrospective cohort study performed at Rutgers Robert Wood Johnson University Hospital in New Brunswick, New Jersey, USA. All adult patients (aged $\geq 18$ years) who presented to the emergency department between January 1, 2020 and April 30, 2020 and had a confirmed COVID-19 diagnosis by PCR nasopharyngeal swab testing were included. Patients who did not have a positive test in the hospital were excluded from the analysis. Data were extracted from patients' medical records using a standardised abstraction format. GI manifestations were defined as nausea, vomiting, abdominal pain, or diarrhoea. LI was defined as an elevation in liver enzymes (aspartate transaminase [AST] $>45$, alanine aminotransferase [ALT] $>46$, alkaline phosphatase $>120$, or total bilirubin >0.8). ${ }^{16}$ Patients who had $\mathrm{Gl}$ manifestaitons or liver abnormalities on admission were included in the analysis. The primary clinical outcome was the impact of $\mathrm{Gl}$ findings and LI on in-hospital mortality. Secondary outcomes were length of stay in hospital and need for intensive care unit (ICU) level care.

\section{Statistical Analysis}

Statistical analysis was conducted using the SAS 9.4 programme. The categorical variables were presented as frequency with percentages, and continuous variables as means with standard deviations. Categorical and continuous variables to compare patients with $\mathrm{Gl}$ symptoms versus patients without $\mathrm{Gl}$ symptoms were made by using chi-square and student t-test. All odds ratios (OR) with 95\% confidence intervals $(\mathrm{Cl})$ were calculated by using logistic regression models adjusting for demographics including age, sex, race, clinical characteristics, and medical comorbidities according to the charlson comorbidity index. A $\mathrm{p}$ value $<0.05$ was considered statistically significant.

\section{RESULTS}

A total of 800 patients' records were reviewed manually, of which 539 patients had presence of SARS-CoV-2 confirmed in the authors' hospital and were included in the study. Demographics of the entire cohort are described in Table 1. The mean age was 60.48 years, average BMI was 28.79 , and $56.2 \%$ of patients were male; $34.2 \%$ of patients were Caucasian, $12.6 \%$ African American, and $24.9 \%$ Hispanic.

Overall mortality was 97/539 (18\%). At least one digestive complaint on admission was seen in 31\% of patients. At presentation, $17.6 \%$ had diarrhoea, $14.4 \%$ had nausea, $10.3 \%$ had vomiting, and $5.9 \%$ had abdominal pain. Of the entire cohort, $64.0 \%$ had LI during hospitalisation, and LI was present in $88.7 \%$ of those who died (Table 1).

In multivariate analysis, Gl manifestations were associated with decreased risk of mortality (OR: 0.46; 95\% Cl: 0.24-0.90; $p=0.0020$ ). GI symptoms did not impact length of stay, 5.06 days for patients with $\mathrm{Gl}$ symptoms versus 4.74 in patients without (OR: 1.01; 95\% Cl: 0.96-1.07; $\mathrm{p}=0.43$ ), or need for ICU (OR: 0.97; 95\% Cl: 0.601.58; $p=0.9076$ ) (Table 2). In multivariate analysis, LI was associated with increased mortality (OR: 8.60; 95\% Cl: 3.49-21.15; $p<0.0001$ ), need for ICU (OR: 10.94; 95\% Cl: 4.07-29.45; $\mathrm{p}<0.0001$ ), and length of stay 5.87 days versus 3.01 days (OR: 1.23; 95\% Cl: 1.14-1.32; $\mathrm{p}<0.0001)$.

The impact of COVID-19 on LI and trend of liver function tests are documented in Table 3. AST and ALT were elevated on admission, with mean values of $68.8 \mathrm{IU} / \mathrm{L}$ and $50.2 \mathrm{IU} / \mathrm{L}$, respectively. On admission, $44.0 \%$ had abnormal AST and $27.1 \%$ had abnormal ALT. At peak values during hospitalisation, $55.5 \%$ had abnormal AST and 39.9\% had abnormal ALT. 
Table 1: Demographics and clinical characteristics.

\begin{tabular}{|c|c|}
\hline Patient characteristics & Patients $n=539$ \\
\hline Age, mean (SD) & $60.48(18.7)$ \\
\hline BMI, mean (SD) & $28.79(7.1)$ \\
\hline \multicolumn{2}{|l|}{ Sex, n (\%) } \\
\hline Male & $303(56.2)$ \\
\hline Female & $236(43.8)$ \\
\hline \multicolumn{2}{|l|}{ Race/ethnicity, n (\%) } \\
\hline Caucasian & $185(34.2)$ \\
\hline African American & $68(12.6)$ \\
\hline Hispanic & $134(24.9)$ \\
\hline South Asian & $30(5.6)$ \\
\hline East Asian & $38(7.1)$ \\
\hline Other & $84(15.6)$ \\
\hline \multicolumn{2}{|l|}{ Type of stay, n (\%) } \\
\hline Triage & $45(8.4)$ \\
\hline Observation & $72(13.4)$ \\
\hline Inpatient & $422(78.3)$ \\
\hline \multicolumn{2}{|c|}{ Gastrointestinal symptoms, n (\%) } \\
\hline Nausea & $78(14.5)$ \\
\hline Vomiting & $56(10.4)$ \\
\hline Diarrhoea & $95(17.6)$ \\
\hline Abdominal pain & $32(5.9)$ \\
\hline
\end{tabular}

Table 2: Impact of gastrointestinal manifestations and liver injury on mortality and clinical outcomes.

\begin{tabular}{|c|c|c|c|c|}
\hline Outcomes & GI symptoms ( $n=165)$ & $\begin{array}{l}\text { No Gl symptoms } \\
(n=374)\end{array}$ & OR $(95 \% \mathrm{Cl})$ & p value \\
\hline Mortality, n (\%) & $17(10.3)$ & $80(21.4)$ & $0.46(0.24-0.90)$ & 0.0020 \\
\hline $\begin{array}{l}\text { Length of hospital } \\
\text { stay, mean (SD) }\end{array}$ & $5 . .06(4.6)$ & $4.74(4.2)$ & $1.01(0.96-1.07)$ & 0.4302 \\
\hline ICU, n (\%) & $28(17)$ & $65(17.4)$ & $0.90(0.51-1.59)$ & 0.9076 \\
\hline Outcomes & Liver injury $(n=345)$ & No liver injury $(n=194)$ & OR $(95 \% \mathrm{Cl})$ & $p$ value \\
\hline Mortality, n (\%) & $86(24.9)$ & $11(5.7)$ & $8.60(3.49-21.15)$ & $<0.0001$ \\
\hline $\begin{array}{l}\text { Length of hospital } \\
\text { stay, mean (SD) }\end{array}$ & $5.87(4.5)$ & $3.01(3.3)$ & $1.23(1.14-1.32)$ & $<0.0001$ \\
\hline ICU, n (\%) & $86(24.9)$ & $7(3.6)$ & $10.94(4.07-29.45)$ & $<0.0001$ \\
\hline
\end{tabular}

Cl: confidence interval; GI: gastrointesinal; ICU: intensive care unit; OR: odds ratio; SD: standard deviation. 
Table 3: Trend of liver laboratory test.

\begin{tabular}{|l|l|l|l|l|l|l|}
\hline & $\begin{array}{l}\text { Mean initial } \\
(\text { SD) }\end{array}$ & $\begin{array}{l}\text { Abnormal } \\
\text { initial* } \mathbf{n}(\%)\end{array}$ & $\begin{array}{l}\text { Mean peak } \\
(\mathrm{SD})\end{array}$ & $\begin{array}{l}\text { Abnormal } \\
\text { peak }^{+}, \mathbf{n}(\%)\end{array}$ & $\begin{array}{l}\text { Mean trough } \\
(\mathrm{SD})\end{array}$ & $\begin{array}{l}\text { Abnormal } \\
\text { trough }^{+}, \mathbf{n}(\%)\end{array}$ \\
\hline AST (IU/L) & $68.8(163.2)$ & $237(44)$ & $160.4(590.3)$ & $299(55.5)$ & N/A & N/A \\
\hline ALT (IU/L) & $50.2(96.8)$ & $146(27.1)$ & $110.96(399.1)$ & $215(39.9)$ & N/A & N/A \\
\hline ALP (IU/L) & $83.0(51.9)$ & $54(10.0)$ & $97.28(64.2)$ & $101(18.7)$ & N/A & N/A \\
\hline Albumin (g/dL) & $3.6(1.3)$ & $245(45.5)$ & N/A & N/A & $3.1(0.8)$ & $411(76.3)$ \\
\hline Bilirubin (mg/dL) & $0.5(0.3)$ & $61(11.3)$ & N/A & N/A & N/A & N/A \\
\hline
\end{tabular}

*initial $=$ on admission.

†= peak/trough during hospitalisation

ALP: alkaline phosphatase; ALT: alanine aminotransferase; AST: aspartate transaminase; SD: standard deviation.

\section{DISCUSSION}

\section{Gastrointestinal Manifestations}

The results of this study indicate that $\mathrm{Gl}$ symptoms are inversely associated with the risk of mortality. In total, $30.6 \%$ of patients in the cohort had $\mathrm{Gl}$ symptoms on admission. In the patients who died, $10.3 \%$ had Gl symptoms, whereas Gl symptoms were present in $21.4 \%$ of those who survived, for an OR of 0.46 .

Published literature has provided mixed results. A study published in China reported that digestive symptoms were more pronounced as the severity of COVID-19 increased..$^{13}$ A meta-analysis confirmed similar results in that patients with severe or critical disease were more likely to have $\mathrm{Gl}$ symptoms. However, the risk of severe disease was not associated with Gl symptomology. ${ }^{17}$ No differences in mortality or length of stay were reported between the two groups. Hajifathalian et $\mathrm{al}^{15}$ showed that the presence of $\mathrm{Gl}$ symptoms on initial presentation had no significant effect on mortality in a cohort of patients in the USA. ${ }^{15}$ In addition, these patients had lower rates of death and ICU admissions. Further, Nobel et al. ${ }^{18}$ demonstrated that GI symptoms were associated with longer duration of illness and a trend toward lower ICU admission rates and lower mortality after a median 8-day follow-up in the outpatient setting. These findings may suggest that patients with digestive symptoms are less likely to have respiratory symptoms. Conversely, in a recent systematic review and meta analysis of COVID-19 patients by Tariq et al.,19 21 studies, totalling 4,982 patients, reported mortality as an outcome in patients with Gl symptomology. Tariq et al..$^{19}$ found that one in five patients report $\mathrm{Gl}$ symptomology and found that patients with GI manifestations had similar overall mortality to those who did not. Furthermore, in a systematic review by Gul et al., ${ }^{20}$ revealed that patients with GI symptoms of COVID-19 did not have an increased risk of mortality but may be at higher risk to develop acute respiratory distress syndrome. Cao et al., ${ }^{21}$ demonstrated that GI symptomology are not associated with severe disease or worse outcomes, but cautioned the presence of SARS-CoV-2 RNA in faeces after attenuation of respiratory symptoms. In addition, previous studies have also demonstrated no further increased risk of COVID-19-associated mortality in patients with inflammatory bowel disease. 22,23

SARS-CoV-2 RNA has previously been identified by several studies in the stool samples of patients with COVID-19. Wu et al. ${ }^{9}$ demonstrated the presence of SARS-CoV-2 RNA in stool samples 
after respiratory samples tested negative and respiratory illness subsided. This could indicate the viability of COVID-19 in the GI tract. It remains to be demonstrated whether pulmonary manifestations and higher resultant mortality rates are 'attenuated' by viral shedding via the GI tract. It should be recongised that symptoms may be under-reported in earlier studies due to incomplete questionaires and may be higher prevalence today due to increasing awareness of $\mathrm{Gl}$ manifestations of COVID-19 and more comprehensive review of systems in recent hospitalisations. Initial data were collected through subjective reports of $\mathrm{Gl}$ symptomology while specific $\mathrm{Gl}$ testing to date has occurred in limited studies, i.e., labarotory testing, imaging, endoscopic evaluation, and histopathology.

\section{Liver Injury}

This study indicates that LI was associated with a significantly increased risk of mortality, need for ICU admission, and length of stay. Of the patients who died, $88.7 \%$ had LI, whereas only $64.0 \%$ of patients had abnormal liver enzymes during hospitalisation. Further, patients had increasing LI during their hospitalisation as they had rising mean elevations in liver enzymes and increased LI (Table 3). Interestingly, mean AST results were higher than ALT values at initial presentation as well as at peak values.

Thirteen studies reported the association between LI and severity of the disease but only reported univariate analysis. ${ }^{14}$ Hajifathalian et al. ${ }^{15}$ performed a multivariate analysis that reported an association between $\mathrm{LI}$ on presentation and a significantly higher risk of ICU admission and death. ${ }^{15}$ Previous studies alongside these findings suggest that liver enzyme abnormalities may portend worse outcomes and increased mortality. In a multicentre retrospective cohort study by Lei et al., ${ }^{24}$ elevated LI, particularly AST, was strongly associated with increased mortality risk and was associtated with decreased lymphocyte count, neutrophilia, and male sex. In a systematic review by Ghoda et al., ${ }^{25} \mathrm{LI}$ correlated with severity of COVID-19 illness.

Liver damage may be ascribed to direct viral infection of hepatocytes and cholangiocytes, mitochondrial injury, drug hepatotoxicity (druginduced LI), immune-mediated inflammation, cytokine-mediated injury, sepsis, and liver failure in patients who are critically ill. ${ }^{26}$ Microthrombi, decreased perfusion, and pneumonia-induced hypoxaemia may also contribute to LI. ${ }^{27}$ Autopsy results from patients with the 2002-2003 SARSCoV demonstrated that SARS-CoV was directly able to induce cytopathic damage to hepatocytes rather than damage elicited by cytokine damage secondary to sepsis. A recent study by Alqahtani and Schattenberg that overviewed pathological findings of liver biopsy specimens of patients with COVID-19 demonstrated inflammation in the lobular and portal regions of the liver and microvesicular steatosis, both of which are nonspecific injuries to the liver and may be secondary to sepsis or drug-induced LI. ${ }^{28}$ Caveat to elevations in transaminases, specifically AST, is the possibility of muscle injury. ${ }^{29}$

Literature demonstrated higher presence of ACE2R expression in cholangiocytes in comparison with hepatocytes, and as a result, cholangiocytes may be responsible for susseptability and injury of the hepatobiliary system secondary to COVID-19.30 This may include interrupting liver physiology including, but not limited to, adaptive immune response mechanism, regeneration, and basal function, i.e., dysregulation of formation and transport of bile. ${ }^{28}$ Three studies that analysed a cholestaticspecific marker, gamma glutamyl transferase, support hepatobiliary disruption mediated by COVID-19..$^{31-33}$ As current COVID-19 data are limited in breadth, future studies and prospective cohorts should incorporate specific markers, for example gamma glutamyl transferase, to determine and confirm specific ramifications of the viral infection in the hepatobiliary system.

LI data from COVID-19 do not take into account pre-existing liver disease or follow-up of $\mathrm{LI}$ after acute hospitalisation. Further analaysis of patients with pre-existing disease and disease prognosis and outcomes subsequently after COVID-19 infection must be evaluated in future research. Limitations of this study include that it's a retrospective study and single-centre analysis. This analysis included a relatively small cohort of patients but included a diverse population.

\section{CONCLUSION}

GI findings and LI are common in hospitalised patients with COVID-19. GI symptoms may 
inversely correlate with mortality, while $\mathrm{LI}$ is

findings along with prospective studies to associated with increased mortality, length determine the long-term implications of of stay, and ICU admission. Further study COVID-19 in the GI and hepatobiliary systems.

is needed on the mechanisms of these

\section{References}

1. Cascella $\mathrm{M}$ et al. Features, evaluation, and treatment of coronavirus. In: StatPearls [Internet]. 2020. Treasure Island (FL): StatPearls Publishing.

2. Balkhair AA. COVID-19 pandemic: a new chapter in the history of infectious diseases. Oman Med J. 2020;35(2):e123.

3. World Health Organization (WHO). Coronavirus disease (COVID-19) pan-demic. 2020. Available at: https://www.who.int/emergencies/ diseases/novel-coronavirus-2019. Last accessed: 26 Jan 2021.

4. Ni W et al. Role of angiotensinconverting enzyme 2 (ACE2) in COVID-19. Crit Care. 2020;24(1):422.

5. Lei J et al. Nsp3 of coronaviruses: structures and functions of a large multi-domain protein. Antiviral Res. 2018;149:58-74.

6. Cole-Jeffrey CT et al. ACE2 and microbiota: emerging targets for cardio-pulmonary disease therapy. J Cardiovasc Pharmacol. 2015;66(6):540-50.

7. Fliser $D$ et al.; EUropean Trial on Olmesartan and Pravastatin in Inflamma-tion and Atherosclerosis (EUTOPIA) Investigators. Antiinflammatory effects of angiotensin II subtype 1 receptor blockade in hypertensive patients with microinflammation. Circulation. 2004;110(9):1103-7.

8. $\mathrm{Xu} \mathrm{J}$ et al. Digestive symptoms of COVID-19 and expression of ACE2 in digestive tract organs. Cell Death Discov. 2020;6(1):1-8.

9. Wang $D$ et al. Clinical characteristics of 138 hospitalized patients with 2019 novel coronavirus-infected pneumonia in Wuhan, China. JAMA. 2020;323(11):1061-9.

10. Pan $L$ et al. Clinical characteristics of COVID-19 patients with digestive symptoms in Hubei, China: a descriptive, crosssectional, multicenter study. Am J Gastroenterol. 2020;115(5):766-73.

11. Villapol S. Gastrointestinal symptoms associated with COVID-19: impact on the gut microbiome. Transl Res. 2020;226:57-69.
12. Rokkas T. Gastrointestinal involvement in COVID-19: a systematic review and meta-analysis. Ann Gastroenterol. 2020;33(4):355-65.

13. Cheung KS et al. Gastrointestinal manifestations of SARS-CoV-2 infection and virus load in fecal samples from a Hong Kong cohort: systematic re-view and meta-analysis. Gastroenterology. 2020;159(1):81-95.

14. Sultan $S$ et al. AGA institute rapid review of the gastrointestinal and liver manifestations of COVID-19, meta-analysis of international data, and recommendations for the consultative management of patients with COVID-19. Gastroenterology. 2020;159(1):320-34.e27.

15. Hajifathalian K et al. Gastrointestinal and hepatic manifestations of 2019 novel coronavirus disease in a large cohort of infected patients from New York: clinical implications. Gastroenterology. 2020;159(3):113740.e2.

16. Malakouti $\mathrm{M}$ et al. Elevated liver enzymes in asymptomatic patients - what should i do? J Clin Transl Hepatol. 2017;5(4):394-403.

17. Mao R et al. Manifestations and prognosis of gastrointestinal and liver involvement in patients with COVID-19: a systematic review and meta-analysis. Lancet Gastroenterol Hepatol. 2020;5(7):667-78.

18. Nobel YR et al. Gastrointestinal symptoms and coronavirus disease 2019: a case-control study from the United States. Gastroenterology. 2020;159(1):373-5.e2.

19. Tariq $R$ et al. Prevalence and mortality of COVID-19 patients with gastrointestinal symptoms: a systematic review and meta-analysis. Mayo Clin Proc. 2020;95(8):1632-48.

20. Gul F et al. Meta-analysis of outcomes of patients with COVID-19 infection with versus without gastrointestinal symptoms. Proc (Bayl Univ Med Cent). 2020;33(3):366-9.

21. Cao $\mathrm{C}$ et al. Clinical features and outcomes of COVID-19 patients with gastrointestinal symptoms. Crit Care. 2020;DOI:10.1186/s13054-02003034-x. [Epub ahead of print].

22. Taxonera $\mathrm{C}$ et al. 2019 novel coronavirus disease (COVID-19) in patients with inflammatory bowel diseases. Aliment Pharmacol Ther. 2020;52(2):276-83.

23. Bezzio $C$ et al. Outcomes of COVID-19 in 79 patients with IBD in Italy: an IGIBD study. Gut. 2020;69(7):1213-7.

24. Lei $\mathrm{F}$ et al. Longitudinal association between markers of liver injury and mortality in COVID-19 in China. Hepatology. 2020;72(2):389-98.

25. Ghoda A, Ghoda M. Liver injury in COVID-19 infection: a systematic review. Cureus. 2020;12(7):e9487.

26. Li J, Fan J-G. Characteristics and mechanism of liver injury in 2019 corona-virus disease. J Clin Transl Hepatol. 2020;8(1):13-7.

27. Pawlotsky J-M. COVID-19 and the liver-related deaths to come. Nat Rev Gastroenterol Hepatol. 2020;17(9):1-3.

28. Alqahtani SA, Schattenberg JM. Liver injury in COVID-19: the current evi-dence. United European Gastroenterol J. 2020;8(5):509-19.

29. Jin $M$, Tong Q. Rhabdomyolysis as potential late complication associated with COVID-19 Emerg Infect Dis. 2020;26(7):1618-20.

30. Chai $X$ et al. Specific ACE2 expression in cholangiocytes may cause liver damage after 2019-nCoV infection. bioRxiv. 2020;DOI:10.1101/2020.02.03. 931766. [Preprint].

31. Fan $Z$ et al. Clinical features of COVID-19-related liver functional abnormality. Clin Gastroenterol Hepatol. 2020;18(7):1561-6.

32. Zhang $C$ et al. Liver injury in COVID-19: management and challenges. Lancet Gastroenterol Hepatol. 2020;5(5):428-30.

33. $\mathrm{Xu} L$ et al. Liver injury during highly pathogenic human coronavirus infections. Liver Int Off J Int Assoc Study Liver. 2020;40(5):998-1004. 